\title{
Multi-Seam Tracking with a Portable Robotic Welding System in Unstructured Environments
}

\author{
Shuangfei $\mathrm{Yu}^{1}$, Yisheng Guan ${ }^{*}$, Zhi Yang ${ }^{1}$, Chutian \\ $\mathrm{Liu}^{1}$, Jiacheng $\mathrm{Hu}^{1}$, Jie Hong ${ }^{3}$, Haifei Zhu ${ }^{1}$ and Tao \\ Zhang $^{1,2^{*}}$
}

${ }^{1 *}$ School of Electro-mechanical Engineering, Guangdong University of Technology, Guangzhou, 510006, China. $2^{*}$ Department of Physical Intelligence, Max-Planck Institute for Intelligent Systems, Stuttgart, 70569, Germany.

${ }^{3}$ School of Engineering and Computer Science, Australian National University, Canberra, ACT 2601, Australia.

*Corresponding author(s). E-mail(s): ysguan@gdut.edu.cn; tzhang@gdut.edu.cn;

Contributing authors: yushuangfei163@163.com;

\begin{abstract}
Most welding manufacturing of the heavy industry, such as shipbuilding and construction, is carried out in an unstructured workspace. The term Unstructured indicates the production environment is irregular, changeable and without model. In this case, the changeable workpiece position, workpiece shape, environmental background, and environmental illumination should be carefully considered. Because of such complicated characteristics, the welding is currently being relied on the manual operation, resulting in high cost, low efficiency and quality. This work proposes a portable robotic welding system and a novel seam tracking method. Compared to existing methods, it can cope with more complex general spatial curve weld. Firstly, the tracking pose of the robot is modeled by a proposed dual-sequence tracking strategy. On this basis, the working parameters can be adjusted to avoid robot-workpiece collision around the workpiece corners during the tracking process. By associating the forward direction of the welding torch with the viewpoint direction of the camera,
\end{abstract}


it solves the problem that the weld feature points are prone to be lost in the tracking process by conventional methods. Point cloud registration is adopted to globally locate the multi-segment welds in the workpiece, since the system deployment location is not fixed. Various experiments on single or multiple welds under different environmental conditions show that even if the robot is deployed in different positions, it can reach the starting point of the weld smoothly and accurately track along the welds.

Keywords: robotic welding, visual servo, multi-seam tracking, unstructured enviroments

\section{Introduction}

As a basic fabrication method in joining materials, usually metals or thermoplastics, welding plays an irreplaceable role in modern manufacturing, especially in the heavy industry. It is well known that up to $70 \%$ of the world steel production is used for the manufacturing of welded products, structures and equipment. Because of this, it is still one of the leading technological processes in the world economy [1]. It is employed in the construction of numerous industrial sectors, such as the ship building, railway industry productions, energy sector, machine building, bridge construction, automobile industry, transport, agriculture, petrol industry and even in space technologies. In many cases, welding is the only possible and most effective method of building unassembled joints and resource retaining structures, approximating the optimal shape.

However, in stark contrast, most of the welding work in unstructured environments still relies on manual work. According to statistics, more than $90 \%$ of the welding work is still done manually and this probability is even higher in the heavy industry $[2,3]$. This production method has inherent drawbacks, for instance, the human work is costly, and the workers may suffer from health problems owing to the unavoidable strong heat radiation, smoke, and arc during the welding process. More importantly, the human work cannot consistently guarantee the welding quality which may influence the product safety, especially in public transport products such as ships, high-speed railways and bridges. Unfortunately, it is pretty difficult to conduct automatic welding in the heavy industry because, different from the structured production lines in traditional workshops, the working environment is often unstructured, including: (i) Workpiece diversity. Unlike the assembly line where every thing is fixed, various structural parts with different shapes and sizes in the heavy industry are assembled on-site. (ii) Workplace variability. Most welding work is carried out in the outdoor environment, requiring the welding platform to have excellent mobility, which is hard to achieve with conventional cumbersome industrial robots. 
Robotic welding is now a major development in the manufacturing industry due to the significant advantages of welding robots in improving efficiency, optimizing quality, and improving labor conditions. The above dilemma drives the research of mobile-robotic welding system for the purpose of flexible deployment in the heavy industry. With respect to the mechanical design, the current mobile-robotic welding systems are mainly divided into three categories: the rail-based system [4-7], the welding carriage system [8-10] and hanging basket based system. The first, including the well-known commercail product, IGM welding robot, is limited to the applications in structured environments. For example, a welding system based on a gantry mechanism with a guide rail is demonstrated to weld large containers [6]. The second is convenient to deploy because of its small size, however, it cannot weld complex spatially curved welds. This kind of system, including the representative commercial products, such as LIZARD from ITM [11], Wel-Handy Multi Next from Koike Aronson [12], is usually driven by wheels [8] or portable rails [9, 13]. During its working process, the carriage moves along the welds to achieve continuous tracking and welding. One drawback is that the end-effector is attached with the carriage, hence its working space is limited, making it unsuitable for large and complex surfaces. In respect of the third, there are relatively few mature cases, such as a fixed six-axis robot called DANDY is used to weld single-hull structures by Daewoo Shipbuilding and Marine Engineering in Geojie, Korea [14]. An overhead gantry crane installed on the ceiling of the manufacturing factory moves DANDY between welding locations. This has been discarded for now because of its predictable unwieldiness. In conclusion, the mobile-robotic system for welding in the heavy industry still has drawbacks to be solved.

Different from traditional methods, such as teaching-reproduction or offline programming, the mobile-robotic welding system faces two key issues for spatial multi-weld tracking: the global positioning of multi-section welds, and weld seam tracking problem. In order to achieve continuous tracking of multiple welds in a structural part, the entire workpiece must be positioned to guide the robot to transition between different welds. However, due to the influence of various factors such as illumination and background in the unstructured environment, the overall identification and positioning of the workpiece is challenging. Paper [15] propose the idea of extracting all detectable weld seams from multi-view RGB images and then filtering and merging them in the corresponding RGB-D images to detect multiple weld seams in the workpiece. However, it does not consider the influence of environmental factors on object edge extraction, and regard the detected edge lines as welds without difference.

For the second key issue, robotic weld tracking refers to the automatic welding operation along the weld seams with the help of sensors without human intervention. At present, most of the current research is simply based on a single continuous plenary weld [16-25], while the problem of continuous tracking of multi-segment spatial welds in structural parts is rarely studied. However, there are often multiple weld seams in actual structural parts. To establish a fully automatic robotic welding system, an automated weld seam extraction 
process with accurate perception of the target workpiece is one of the most challenging tasks [15]. In addition, these methods require certain prior information, such as the curve equation of the weld [26] or the nominal path taught in advance [4, 27-30], which limit their application in unstructured environments. For example, in 2013, a method developed in the paper [18] enables the robust identification of narrow weld seams for ferrous materials combined with reliable image matching and triangulation through the use of $2 \mathrm{D}$ homography. This method is only tested for plane welds, and the interference of welding on the RGB image processing cannot be ignored under actual working conditions, which makes its practical application performance questionable. In 2016, paper [17] proposes a tracking algorithm based on online shape shape matching was proposed, which uses the previous groove shape as the template to locate the next groove shape. This method does not require prior information, but can only achieve tracking of planar curves. In 2020, the authors [26] present a tracking method of complex space curve weld, but this method requires a prior knowledge of the mathematical curve equation for the weld and has major limitations.

In summary, the problem of weld seam tracking in unstructured environments is the key to achieving robotic welding work in heavy industry, but little research has been done on this problem up to date. In this presentation, a novel weld tracking method for the portable welding system is proposed, which can realize continuous automatic welding of multiple welds in unstructured environments. The remainder of this work is organized as follows: Section 2 introduces the design of the robotic welding system and the overview of weld tracking algorithm; Section 2.3 describes the designed laser vision sensing system and its calibration method; Section 3 presents the tracking method for complex curved welds and global positioning method for multiple welds; Section 4 illustrates the laboratory tests and results; Section 5 concludes this work.

\section{System Description}

\subsection{System Overview}

The proposed system for mobile-robotic welding in unstructured environments is shown in Fig. 1. It mainly consists of cooperative manipulator (Elfin E05), a robot controller, a vision system, and a host computer (Intel NUC11). The cooperative robot carries the welding torch (Panasonic YD-500GR5) under the motion control signals from the controller. The host computer processes the information captured by the vision sensor and gives commands to robot controller. The sensor is installed around the end of cooperative robot. The robot can be placed on a variety of mobile platforms, including the AGV. Besides, human can choose to carry the cooperative robot to the work site. As shown in the right part of Fig. 1, the mass of the cooperative robot with the end-effector and vision sensor is around $23 \mathrm{~kg}$. 


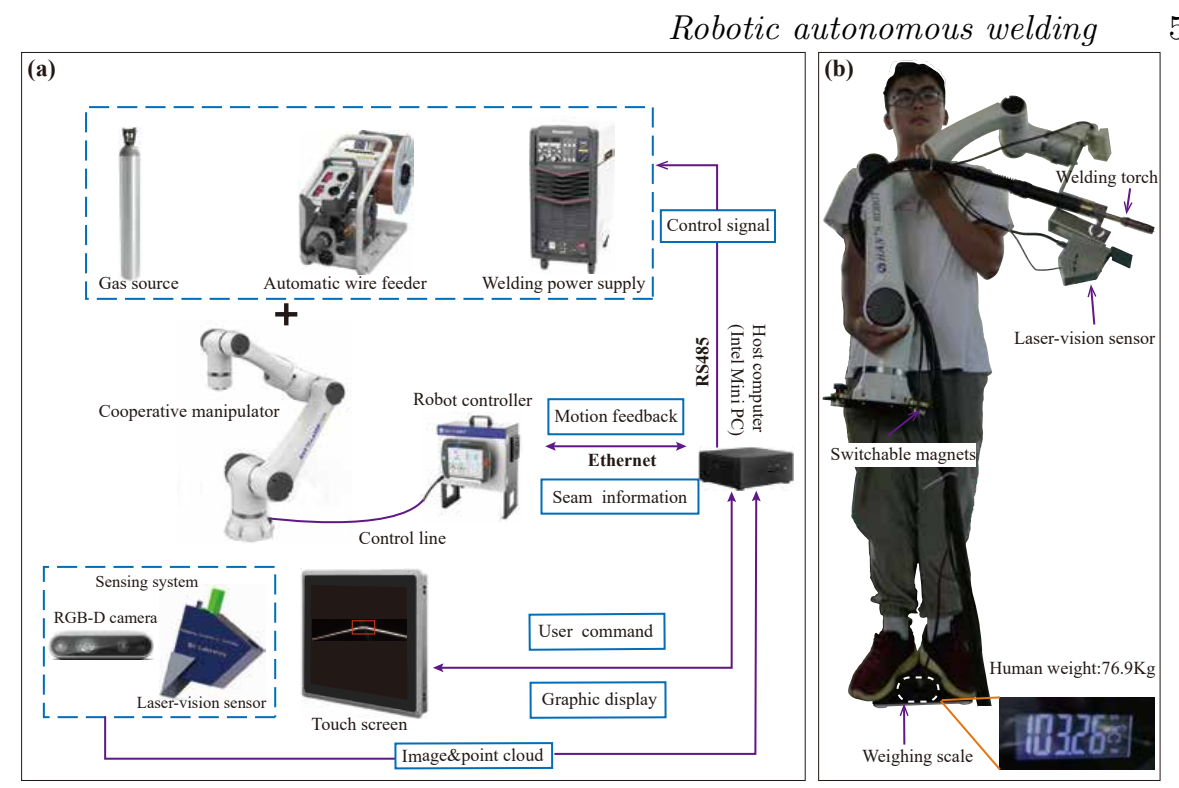

Fig. 1: Overview of the mobile-robotic welding system. (a) System components; (b) Portability demonstration.

The vision system contains two sensors. The first is a RGB-D camera (Realsense D435i), working to roughly locate the workpiece. In order to complete the weld detection, we use the second vision sensor, a self-designed laser vision sensor. Using this sensor, the shape and the position of weld can be accurately detected based on the principle of laser triangle. Further, we establish a reliable imaging model to reconstruct the weld, which will be introduced in Section 2.3.

\subsection{Weld Seam Tracking Algorithm}

The weld seam tracking algorithm of our system is divided into several modules: seam global identification, seam local identification and abnormal monitoring. The whole process is depicted in Fig. 2.

(1) Seam global identification: This process employs the geometry of workpiece to determine spatial distribution of each weld under the robot base coordinate system. This is accomplished by extracting the global characteristics of each weld from the CAD model of the workpiece. As such, we can perform the offline attitude planning of the end-effector and the more accurate positioning of the weld.

(2) Seam local detection: The seam local detection is mainly to use image processing algorithm for feature points extraction of the seam. In particular, we use a KCF tracker [31] combined with the geometric morphological processing to obtain the position of the weld feature points, which is introduced in Section. 3.1.1. The results from the tracker and the geometric morphological processing are fused by Kalman filter to get the final result. 
Robotic autonomous welding

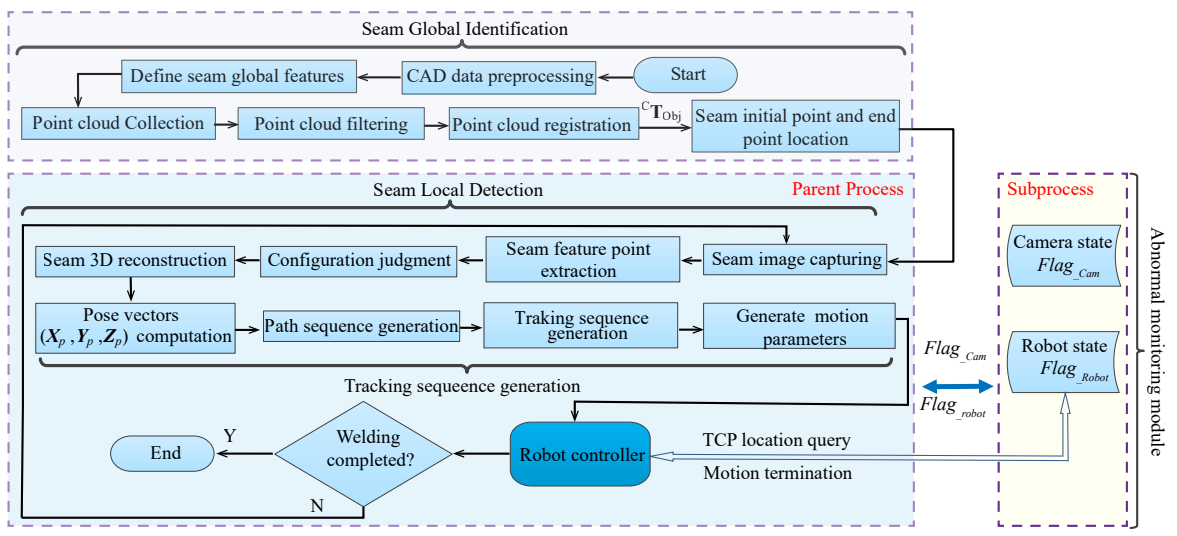

Fig. 2: Weld Seam Tracking Algorithm. The workflow of the proposed weld seam tracking algorithm is provided. There are two main processes: welds global identification and local detection.

(3) Abnormal monitoring: The module monitors two safety indicators:

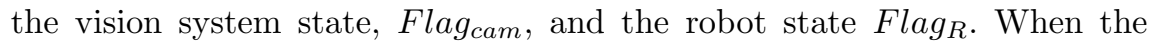
camera stops working(Detected by indicator Flag cam $_{\text {) }}$ ) or the motion position of the cooperative manipulator deviates largely from the expected position(Detected by indicator $F l a g_{R}$ ), the robotic system would stop timely.

\subsection{Laser-Vision Sensing System}

\subsubsection{Mathematical Model of 3D Reconstuction}

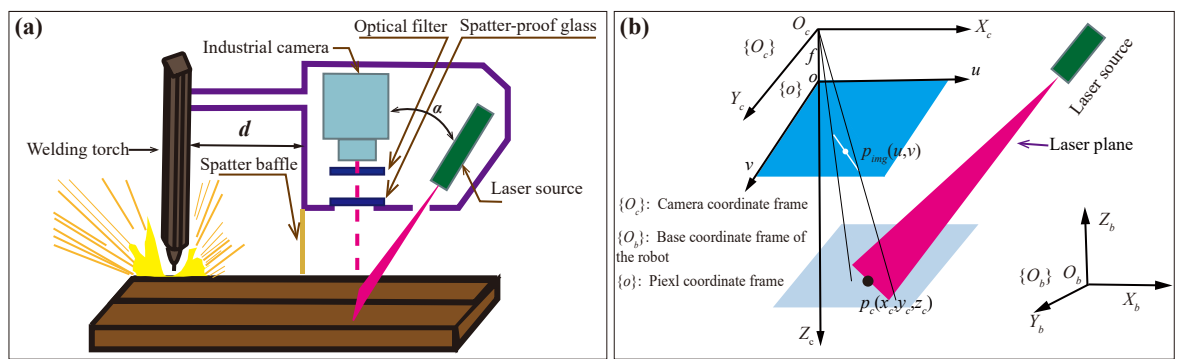

Fig. 3: Laser-vision sensor: diagram of mathematical model. (a) Sensor Configuration; (b) Imaging Model.

The mathematical model of the laser sensor is introduced in this part. The purpose of laser-vision sensor is to obtain the point position $p_{c}\left(x_{c}, y_{c}, z_{c}\right)$ under the camera coordinate via the pixel position $p_{i}(u, v)$ under the image coordinate. The structured light plane equation is fisrtly introduced, then the correction of the image distortion is provided. We first introduce the lens distortion model $[32,33]$ :

$$
\left(\begin{array}{c}
u \\
v
\end{array}\right)=\left(\begin{array}{c}
\widetilde{u}+\widetilde{u}\left(K_{1} r^{2}+K_{2} r^{4}+K_{3} r^{6}\right)+P_{1}\left(r^{2}+2 \widetilde{u}\right)+2 P_{1} \widetilde{u} \widetilde{v} \\
\widetilde{v}+\widetilde{v}\left(K_{1} r^{2}+K_{2} r^{4}+K_{3} r^{6}\right)+2 P_{1} \widetilde{u} \widetilde{v}+P_{2}\left(r^{2}+2 \widetilde{v}\right)
\end{array}\right)
$$


where $(\widetilde{u}, \widetilde{v})$ is the distorted pixel on an image taken by the camera and the corrected pixel is denoted as $(u, v) . K_{1}, K_{2}$ and $K_{3}$ are the radial distortion coefficients, and $P_{1}$ and $P_{2}$ are tangential distortion coefficients. These parameters can be obtained by the camera calibration. Through the above formula, the distorted image can be corrected. As shown in Fig. 3, for a certain point $P_{c}=\left(X_{c} . Y_{c}, Z_{c}\right)^{\mathrm{T}}$ on the laser stripe under the camera coordinate, according to the linear imaging model, the corresponding relationship between the pixel coordinate system and the camera coordinate system is:

$$
\left[\begin{array}{l}
u \\
v \\
1
\end{array}\right]=\left[\begin{array}{ccc}
f_{x} & 0 & u_{0} \\
0 & f_{y} & v_{0} \\
0 & 0 & 1
\end{array}\right]\left[\begin{array}{c}
X_{c} / Z_{c} \\
Y_{c} / Z_{c} \\
1
\end{array}\right]=M_{I}\left[\begin{array}{c}
X_{c} / Z_{c} \\
Y_{c} / Z_{c} \\
1
\end{array}\right]
$$

where $M_{I}$ is the Camera internal parameters. Assuming that the plane equation of the line structured light plane in the camera coordinate system is:

$$
A X_{c}+B Y_{c}+C Z_{c}+D=0
$$

Combining Eq. 2 and 3, we can establish the relationship between the positions of the point on the laser stripe under the pixel coordinate and the camera coordinate:

$$
\left[\begin{array}{ccc}
f_{x} & 0 & u_{0}-u \\
0 & f_{y} & v_{0}-v \\
-A & -B & -C
\end{array}\right]\left[\begin{array}{c}
X_{c} \\
Y_{c} \\
Z_{c}
\end{array}\right]=\left[\begin{array}{c}
0 \\
0 \\
D
\end{array}\right]
$$

Then we can get:

$$
\left\{\begin{array}{l}
X_{c}=-\frac{D f_{y}\left(u-u_{0}\right)}{C f_{y} f_{x}+A f_{y}\left(u-u_{0}\right)+B f_{x}\left(v-v_{0}\right)} \\
Y_{c}=-\frac{D f_{y}\left(v-v_{0}\right)}{C f_{y} f_{x}+A f_{y}\left(u-u_{0}\right)+B f_{x}\left(v-v_{0}\right)} \\
Z_{c}=-\frac{D f_{x} f_{y}}{C f_{y} f_{x}+A f_{y}\left(u-u_{0}\right)+B f_{x}\left(v-v_{0}\right)}
\end{array}\right.
$$

where $f_{x}$ and $f_{y}$ are the focal length parameters of the camera. In this way, We can obtain $P_{c}=\left(X_{c}, Y_{c}, Z_{c}\right)^{\mathrm{T}}$ on the laser stripe from the pixel point $P_{i m g}=(u, v)$ of the image via Eq. (5). In order to guide the robot to work, we still need to convert $P_{c}$ from the camera coordinate to the robot base coordinate:

$$
{ }^{b} P={ }^{b} T_{t} \cdot{ }^{t} T_{c} \cdot{ }^{c} P
$$

where ${ }^{t} T_{C}$ is the pose of the camera coordinate system with respect to the robot tool coordinate system, and can be obtained by the hand eye calibration. ${ }^{b} T_{\text {tool }}$ is the pose of the robot tool coordinate system in the robot base coordinate system, which can be read from the robot controller in real time. Through Eq. (5) and (6), we can calculate $P_{b}$ in the robot base coordinate system from any point on the image $P_{i m g}$. 


\subsubsection{Calibration of Laser-Vision Sensor}

This subsection introduces the calibration of the coefficients of Eq. (3): $A, B, C$ and $D$. Although there are many related studies on the calibration of laser-vision sensor, the current main problem of laser vision sensor calibration is the demand for the use of complex calibration artefacts and excessive model parameters. For instance, the authors [34] propos a calibration model composed of camera, lens, laser position and other parameters. Two lasers and a special calibration board are used to calibrate the position parameters of the laser. This operation is difficult to implement, since too many model parameters and special calibration boards are difficult to obtain. In a similar work [35], a special serrated stereo target was used to calibrate the hand eye relationship between laser vision sensor and robot. Paper [36] aiming at the problem of tedious calibration process, the author adopted a plane calibration board with special pattern to implement automatic calibration. However, this kind of calibration board is also a customized product, which is difficult to obtain. In this section, we use the most common calibration board for our Laser-vision sensor. Assuming that the coordinates of a point $P$ on the laser strip in the coordinate system of the calibration plate is $P_{w}=\left(X_{w}, Y_{w}, Z_{w}\right)^{T}$, and in the camera coordinate system is $P_{c}=\left(X_{c}, Y_{c}, Z_{c}\right)^{T}$, and the corresponding pixel coordinate is $(u, v) . R$ and $t$ are the external parameters relative to the calibration board. It can verified as:

$$
P_{c}=R \cdot P_{w}+T
$$

then, Eq. (7) can be deduced to:

$$
P_{w}=R^{-1}\left(P_{c}-t\right)=\left[\begin{array}{lll}
r_{11}^{\prime} & r_{12}^{\prime} & r_{13}^{\prime} \\
r_{21}^{\prime} & r_{22}^{\prime} & r_{23}^{\prime} \\
r_{31}^{\prime} & r_{32}^{\prime} & r_{33}^{\prime}
\end{array}\right]\left[\begin{array}{l}
X_{c}-t_{x} \\
Y_{c}-t_{y} \\
Z_{c}-t_{z}
\end{array}\right]
$$

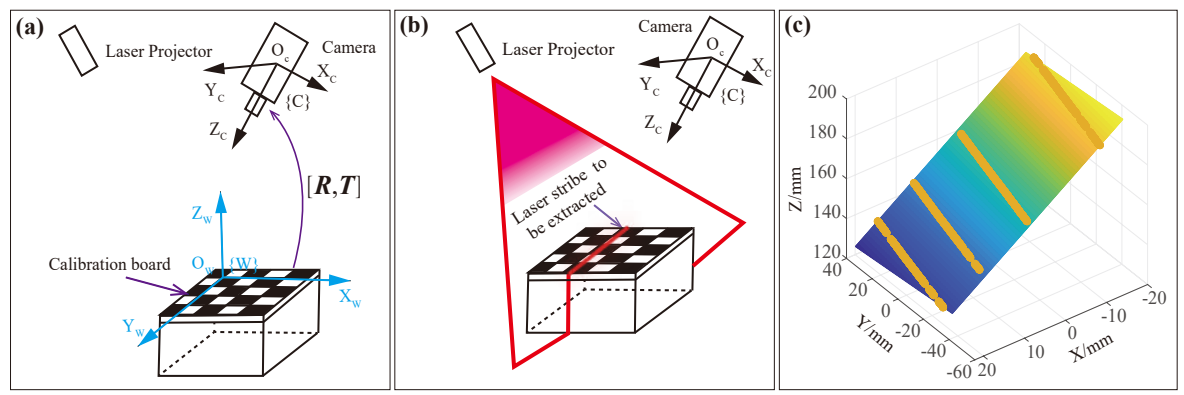

Fig. 4: Laser-vision sensor: calibration. (a) An image of the calibration board is firstly taken by the camera to obtain the relative pose between the calibration board and the camera. (b) Remaining the position and the posture of the camera in (a) and opening the laser projector, we take an image of the laser stripe. (c) Transform the laser stripe point $P_{i m g}$ on the image to point $P_{c}$ under the camera coordinate system. Then, repeat (a) and (b) to obtain multiple points (in yellow color) under the camera coordinate system. Fit the points on these points to a spatial plane. 
where $t=\left[t_{x}, t_{y}, t_{z}\right]^{T}$. Since all points on the laser stripe are located on the plane of the calibration plate, that is, $Z_{w}=0$, then we have:

$$
r_{31}^{\prime}\left(X_{c}-T_{x}\right)+r_{32}^{\prime}\left(Y_{c}-T_{y}\right)+r_{33}^{\prime}\left(Z_{c}-T_{z}\right)=0
$$

combining with (2), we can get:

$$
Z_{c}=\frac{f\left(r_{31}^{\prime} T_{x}+r_{32}^{\prime} T_{y}+r_{33}^{\prime} T_{z}\right)}{r_{31}^{\prime}\left(u-u_{0}\right) d_{x}+r_{32}^{\prime}\left(v-v_{0}\right) d_{y}+r_{33}^{\prime}}
$$

Any point $P_{c}$ in the camera coordinate system can be obtained via Eq. (2) and Eq. (10) from $P_{i m g}$. Keep the camera posture still, change the calibrated posture, and repeat the steps in Fig. 4(a)-(c) to collect multiple images. After converting all collected points to the camera coordinate system, we use SVD method to fit these spatial points into a plane, so we can have coefficients: $A$, $B, C$ and $D$ in Eq. (3).

\section{Seam Tracking Methods}

We have two main cases for seam tracking under unstructured enviroment: single-weld seam tracking and multiple-weld seam tracking. In Section 3.1, we discuss in detail the pose modeling and tracking method for a particular weld seam. On this basis, in Section 3.2, taking ship structural parts as an example, we discuss the continuous tracking method of multi-segment welds by using 3D vision to guide the robot to transition between different welds in the structure.
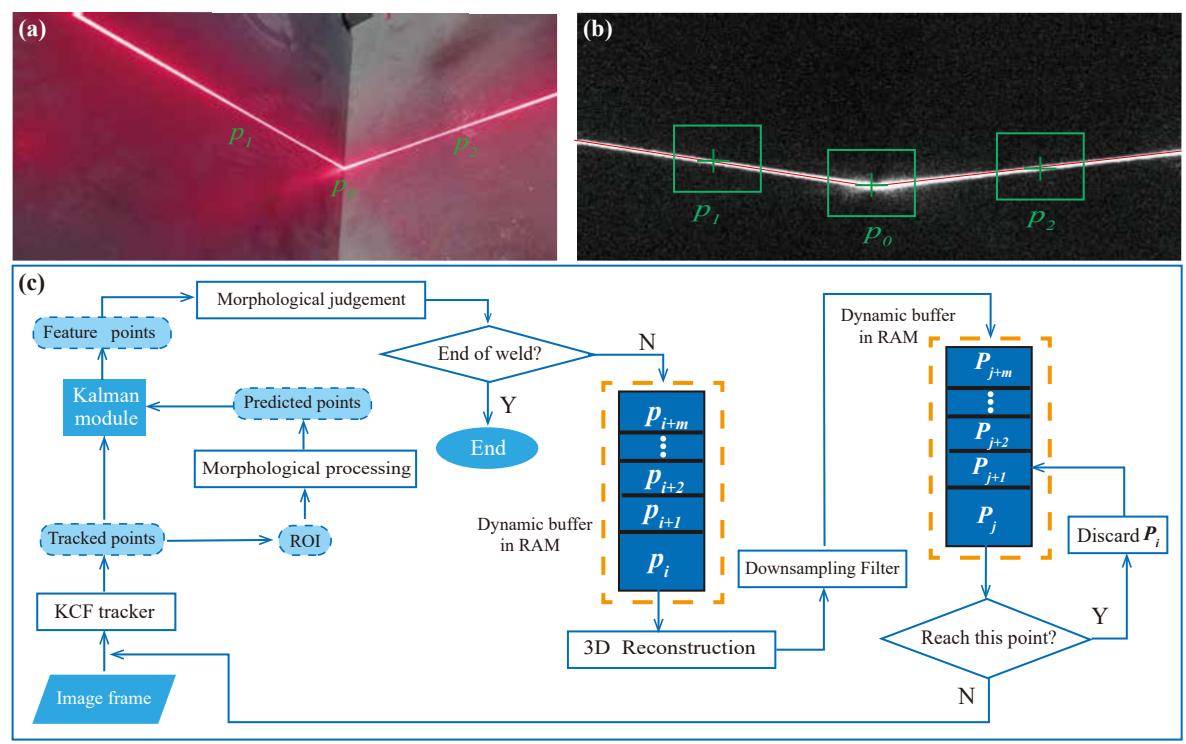

Fig. 5: Weld feature point extraction. (a) Laser stripe on the workpiece; (b) Laser stripe image and the extracted weld feature points on it; (c) Flowchart of the weld feature point extraction. 


\subsection{Single-Weld Seam Tracking}

\subsubsection{Weld Feature Extraction}

The first step of single-weld welding is to extract the weld features via image processing. Overall, the shape of the weld can be obtained via 3D reconstruction of the feature points on the laser stripe of the image captured (See $p_{i}, p_{i, l}$ and $p_{i, r}$ in Fig. $5(\mathrm{~b})$ ). We first extract the center line (see red line in 5 (b)) of the laser strip at the sub-pixel level. Then we find the intersection point $p_{0}(u, v)$, and give $p_{1}$ and $p_{2}$ on the central line which are equally away from $p_{0}(u, v)$.

It is obvious $p_{i}$ in each frame is the target point we need to locate, but it may sometimes fail to get $p_{0}$, due to the interference of spatter and arc during welding. Therefore, we design a robust algorithm in Fig. 5 (c) to find the intersection point of the two lines. Suppose the robot moves to a position, the laser-vision camera captures an image frame. This image frame will be processed by KCF tracker KCF[31] to output trackedpoints. Based on the tracked points, we give the region of interest (ROI), which is the green bounding boxs in Fig. 5 (b). Then we implement Morphological processing to calculate predicted points. Next, using Kalman module, we fuse the tracked points and predicted points to have the final points $p_{0}, p_{1}$ and $p_{2}$ in each frame. Through these three feature points, we can restore the shape of laser stripe, and then use this shape to judge whether the weld reaches the end point. Fig. 5 shows the morphological changes of the laser stripe near the end point (starting point). If we judge that the laser stripe reaches the end of the weld, the algorithm ended. If not, we save $p_{i}$ in the dynamic buffer. Based on $\left\{p_{0,1}, p_{0,2}, p_{0,3}, \ldots\right.$, $\left.p_{0, i}, \ldots\right\}$ in the dynamic buffer, we compute the corresponding robot target points $\left\{P_{1}, P_{2}, P_{3}, \ldots, P_{j}, \ldots\right\}$ under the robot base coordinate system and drive the robot through each target point in turn.

\subsubsection{Seam Trajectory Generation}

When the robot is moving along the weld, due to the difference between the moving speed of the robot and the camera capture frequency, the time steps of the two are not synchronized. If the feature points detected by the camera are directly sent to the robot, huge errors will occur. For example, if the robot moves at speed of $100 \mathrm{~mm} / \mathrm{s}$ and the sensor capture speed is at 10 frames per second, the interval between two images is about $10 \mathrm{~mm}$ which is sparse compared to extracted continuous laser stripe on each image. However, when the robot moves at a slower speed and the camera captures at a faster speed, the feature points obtained from the camera will be very dense with little difference between each other, which will produce a large number of invalid feature points.

Aiming at filtering out the redundant points. Every next point $P_{i+1}$ should satisfy:

$$
d=\left\|P_{i+1}-P_{i}\right\| \geq d_{\text {thres }}
$$


where $d$ is the Euclidean distance between $P_{i+1}$ and $P_{i}$, and usually we set $d_{\text {thres }}$ as $1 \mathrm{~mm}$. The algorithm only processes qualified points. This process is described as downsampling in Fig. 5 (c).

\subsubsection{Tracking Posture Computation}

After we have one computed feature point $p_{i}$ from Section 3.1.2, we can obtain its corresponding weld path point $P_{i}\left(X_{i}, Y_{i}, Z_{i}\right)$ under the base coordinate system of robot. The posture of the weld $\left(X_{p_{i}}, Y_{p_{i}}, Z_{p_{i}}\right)$ under the robot base coordinate system is obtained by firstly curve fitting. For a certain point $P_{i}$ in the weld path detected, the adjacent $2 m$ points are curve-fitted by polynomial. Suppose that the curve equation obtained by fitting is as follows:

$$
\left\{\begin{array}{l}
X_{i}=t \\
Y_{i}=A_{1} t^{3}+B_{1} t^{2}+C_{1} t+D_{1} \\
Z_{i}=A_{2} t^{3}+B_{2} t^{2}+C_{2} t+D_{2}
\end{array}\right.
$$

Afterwards, the tangent vector $\tau_{i}=\left(1, \frac{\partial y}{\partial x}, \frac{\partial z}{\partial x}\right)=\left(1, n_{i, y}, n_{i, z}\right)^{T}$ of $P_{i}$ can be calculated. As shown in Fig. 6 (a), we define the plane $\pi_{i, 1}$ with its normal vector $\tau_{i}$ as the posture plane of $P_{i}$. In order to ensure that the welding torch is always located in the center of the seam, the unit vectors $\boldsymbol{n}_{i, l}$ (from $p_{i}$ to $p_{i, l}$ ) and $\boldsymbol{n}_{i, r}$ (from $p_{i}$ to $p_{i, r}$ ) on both sides of the laser stripe are projected onto the plane $\pi_{i, 1}$ to obtain $\dot{\boldsymbol{n}}_{i, l}$ and $\boldsymbol{n}_{i, r}$, respectively:

$$
\left\{\begin{array}{l}
\dot{\boldsymbol{n}}_{i, l}=\boldsymbol{n}_{i, l}-\operatorname{proj}_{\boldsymbol{\tau}_{i}}\left(\boldsymbol{n}_{i, l}\right) \\
\dot{\boldsymbol{n}}_{i, r}=\boldsymbol{n}_{i, r}-\operatorname{proj}_{\boldsymbol{\tau}_{i}}\left(\boldsymbol{n}_{i, r}\right) \\
\boldsymbol{Z}_{p_{i}}=\dot{\boldsymbol{n}}_{i, l}+\dot{\boldsymbol{n}}_{i, r}
\end{array}\right.
$$

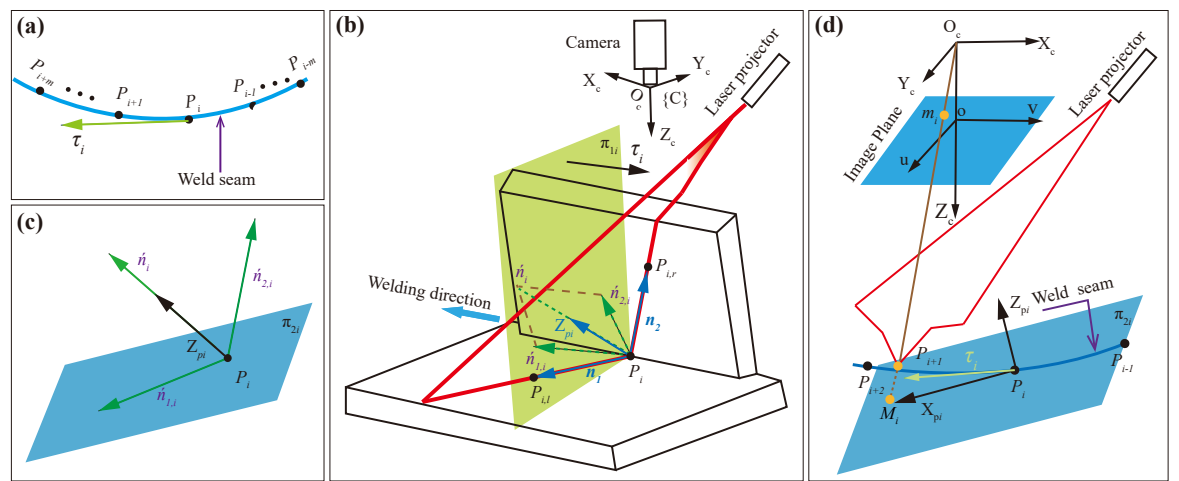

Fig. 6: Tracking posture computation. (a) Find the tangent vector $\tau_{i}$ of the $i$ th path point $P_{i}$; (b) Construct the normal vector $Z_{p}$ of the path point $P_{i}$ from the feature points $p_{0}, p_{1}$ and $p_{2} ;(\mathbf{c})$ Construct the path point tangent plane $\pi_{2 i}$ from $Z_{p} ;$ (d) Calculate the forward direction $X_{p}$ of the welding torch. 

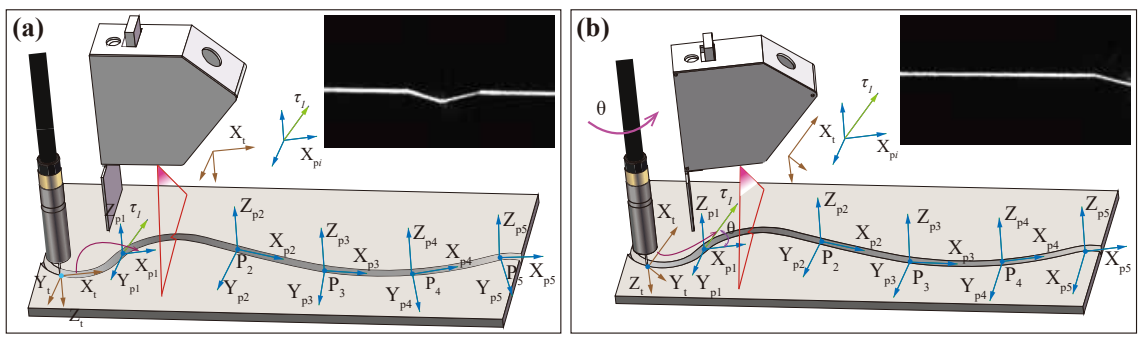

Fig. 7: Comparison of different forward directions in normal tracking attitude with conventional methods $[26,27]$. The traditional method defines the tangential direction $\tau_{i}$ of the path point as the forward direction of the torch, which is easy to cause the loss of the weld feature point. (a) Forward direction defined by our method. (b) Forward direction defined by traditional methods.

Where $\operatorname{proj}_{\boldsymbol{\tau}_{i}}\left(\boldsymbol{n}_{i, l}\right)$ represents the projection vector of $\boldsymbol{n}_{i, l}$ on $\boldsymbol{\tau}_{i}$

$$
\operatorname{proj}_{\boldsymbol{\tau}_{i}}\left(\boldsymbol{n}_{i, l}\right)=\frac{\boldsymbol{\tau}_{i} \cdot \boldsymbol{n}_{i, l}}{\left\|\boldsymbol{n}_{i}\right\|^{2}}
$$

and the rest is the same. The vector $\boldsymbol{Z}_{p_{i}}$ is the direction of the angular bisector between $\dot{\boldsymbol{n}}_{i, l}$ and $\dot{\boldsymbol{n}}_{i, r}$.

In order to define the forward direction of the tracking, we introduce the plane $\pi_{i, 2}$ which is the plane passing through the point $P_{i}$ with $\boldsymbol{Z}_{p_{i}}$ being the normal vector. In order to ensure that the feature point of the weld seam is always within the camera's field of view during the tracking process, we define the line $P_{i+1} O_{c}$ which is between feature point $P_{i+1}$ and the origin of the camera coordinate system $O_{c}$. The intersection point of the extended line of $P_{i+1} O_{c}$ and the plane $\pi_{i, 3}$ is $M_{i}$. We define the unit vector from the current weld feature point $P_{i}$ to $M_{i}$ as the forward direction vector $\boldsymbol{X}_{p_{i}}$ :

$$
\boldsymbol{X}_{p_{i}}=\frac{M_{i}-P_{i}}{\left\|M_{i}-P_{i}\right\|}
$$

Another direction vector $Y_{p_{i}}$ can be obtained via the right-hand rule, i.e., $Y_{p_{i}}=Z_{p_{i}} \times X_{p_{i}}$. Through the above steps, we construct a local posture coordinate system ${ }^{B} \breve{T}^{i}=\left(X_{p_{i}}, Y_{p_{i}}, Z_{p_{i}}\right)$ representing its attitude at each point $P_{i}$. Compared with traditional methods [26, 27], the advantage of our tracking posture calculation is that it can better deal with the complex largecurvature welds adopting single laser stripe. In particular, the robot goes to $P_{i+1}$ while making its orientation pointing to $M_{i}$ rather than $P_{i+1}$, such that the laser-vision sensor would not lost the tracking of the curved seam, see Fig. 7. 


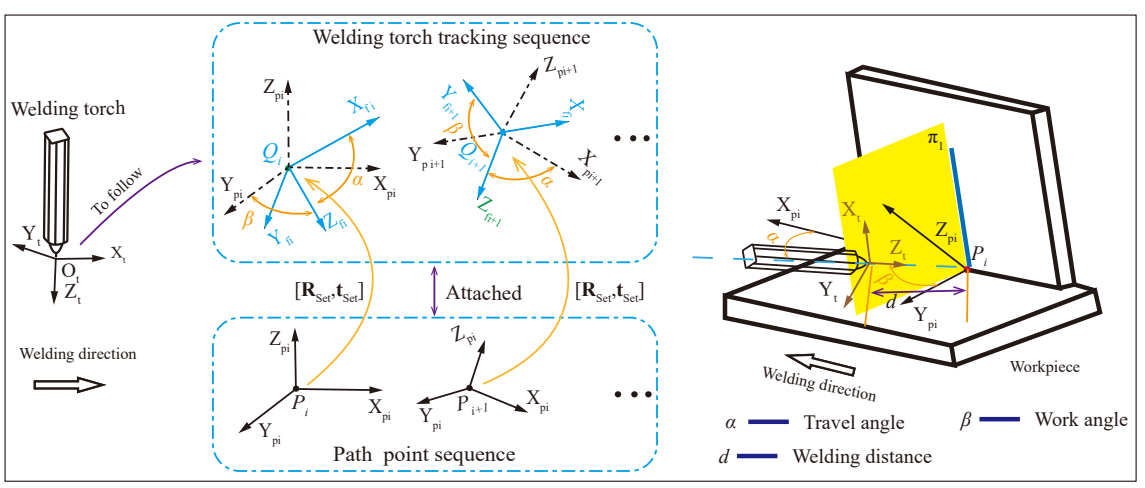

Fig. 8: Dual-sequence tracking strategy for adjusting working parameters.

\subsubsection{Dual-Queue Tracking Strategy}

In actual welding work, in addition to being applied to our dexterous welding method as shown in Fig. 9, the welding pose imposes a significant impact on the mechanical properties of welded joints [37,38]. The Section 3.1.3 introduces how we give the robot the normal posture. However, under many manufacturing scenarios, the welding torch is required not to be in contact with (or be perpendicular to) the weld surface. This asks the robot to properly adjust its posture during the welding. As we know, there are three parameters we can tune: the welding pitch $d$, travel angle $\alpha$, wroking angle $\beta[39,40]$. For each weld path point $P_{i}$, its local attitude is assumed to be ${ }^{B} T^{i}$, then we have:

$$
{ }^{B} \breve{T}^{i}={ }^{B} T^{i} \cdot\left[\begin{array}{cc}
R_{\text {Set }} & t_{\text {Set }} \\
\mathbf{0} & 1
\end{array}\right]={ }^{B} T^{i} \mathcal{T}
$$

where

$$
R_{S e t}=R_{Z}(0) R_{Y}(\beta) R_{X}(\alpha)=\left[\begin{array}{lll}
1 & 0 & 0 \\
0 & 1 & 0 \\
0 & 0 & 1
\end{array}\right]\left[\begin{array}{ccc}
\cos \beta_{i} & 0 & \sin \beta \\
0 & 1 & 0 \\
-\sin \beta & 0 & \cos \beta
\end{array}\right]\left[\begin{array}{ccc}
1 & 0 & 0 \\
0 & \cos \alpha & -\sin \alpha \\
0 & \sin \alpha & \cos \alpha
\end{array}\right]
$$

where ${ }^{B} \breve{T}^{i}$ is the expected pose for the welding torch to be at $P_{i} . \alpha$ and $\beta$ are the angular offset between the tracking coordinate system and the local coordinate system for $P_{i}$. As shown in Fig. 8, by regulating $\mathcal{T}$, we can generate the tracking sequence corresponding to each point in the path sequence oneto-one.

\subsubsection{Cooperative Transformation of Translation and Rotation Based on PBVS}

We use a mechanism similar to Position Based Visual Servo (PBVS) to control the movement of the robot, that is, the robot keeps moving towards the target point $Q_{i}$ in the tracking sequence, once it reaches its position, it will be deleted from the memory and move towards the next target point $Q_{i+1}$. And 
so on until the entire tracking process is completed. In this process, we must ensure that when the robot end-effector reaches each target point, its posture also completes the corresponding transformation. Therefore, the coordinated control between translation motion and rotation motion of robot end-effector is very important.

As shown in Fig. 8 (a), if the welding gun is currently at the $Q_{i}$, we adopt the general velocity motion mode of the robot, whose motion parameter interface is $\left[T_{x}, T_{y}, T_{z}, R_{x}, R_{y}, R_{z}\right]$. The first three variables are the respective speed of the robot moving in $X_{t}, Y_{t}$ and $Z_{t}$ directions while the latter is the speed of the transformation of the pose at each angle of RPY. Let the translation vector between the current point $Q_{i}$ and the next point $Q_{i+1}$ be:

$$
\Delta d=\frac{Q_{i+1}-Q_{i}}{\left\|Q_{i+1}-Q_{i}\right\|}=\left[\Delta t_{x}, \Delta t_{y}, \Delta t_{z}\right]
$$

Suppose the time required for the robot to move from $Q_{i}$ to $Q_{i+1}$ is $t$, then the speed of position movement is

$$
\Delta V_{t}=\frac{\Delta d}{t}=\left[\frac{\Delta t_{x}}{t}, \frac{\Delta t_{y}}{t}, \frac{\Delta t_{z}}{t}\right]=\Delta d \delta
$$

where, $\delta=\frac{1}{t}$ is the speed adjustment of position translation. By adjusting $\delta$, the moving speed of the welding torch can be set in the tracking process.

Assuming that when the welding torch reaches the $Q_{i+1}$ also complete the posture Transformation, it would be preferable to set the posture transformation speed to be consistent with the translation speed $\delta$. Let the pose of $Q_{i}$, $Q_{i+1}$ in the robot base coordinate system is $R_{i}, R_{(i+1)}$, and the transformation matrix between the two is $\Delta R$, then

$$
R_{i+1}=\Delta R R_{i} \quad \Rightarrow \quad \Delta R=R_{i+1} \cdot R_{i}^{-1}=\left[\begin{array}{lll}
r_{11} & r_{12} & r_{13} \\
r_{21} & r_{22} & r_{23} \\
r_{31} & r_{32} & r_{33}
\end{array}\right]
$$

convert $\Delta R$ to RPY angle form

$$
\left\{\begin{array}{l}
\widetilde{R}_{x}=\operatorname{Atan} 2\left(-r_{31}, \sqrt{r_{11}^{2}+r_{21}^{2}}\right) \\
\widetilde{R}_{y}=\operatorname{Atan} 2\left(r_{21}, r_{11}\right) \\
\widetilde{R}_{z}=\operatorname{Atan} 2\left(r_{32}, r_{33}\right)
\end{array}\right.
$$

let $\Delta r=\left[\widetilde{R}_{x}, \widetilde{R}_{y}, \widetilde{R}_{z}\right]$, and its normalized vector is $\Delta \widetilde{r}$, then, the final speed of pose transfermation for robot is $\delta \widetilde{r}=\left[\delta \widetilde{R}_{x}, \delta \widetilde{R}_{y}, \delta \widetilde{R}_{z}\right]=\left[R_{x}, R_{y}, R_{z}\right]$.

In this way, we complete the synchronous control of the robot's translation and rotation posture. Since most robots have speed motion mode, our motion control strategy is also applicable to other robots. 


\subsection{Continuous Tracking of Multi-Weld Seams}

Since our welding system needs to be deployed to different work places, and the posture of each placement is not fixed, it is necessary to guide the robot to the starting point of the weld. For single-weld welding, without using any predefined CAD models, we can easily locate the initial point of weld with the help of the human work. However, under the unstructured workspace, for multipleweld welding, we should loacte initial positions of every weld. Fortunately, CAD model of the workpiece provides us the reliable prior information. Based on the CAD model, we employ Iterative Closest Point (ICP) algorithm [41] to obtain the pose of workpiece ${ }^{C A D} T_{C}$ in camera coordinate system using the the $3 \mathrm{D}$ camera.

\subsubsection{Global Parameters}

Several parameters are defined in this subsubsection for mutliple-weld welding. As shown in Fig. 9 (a) and (b), the welding torch may collide with the workpiece near the corner area if it moves along a certain fixed working attitude during the tracking process. To this end, we adjust theworking attitude of the end-effector by defining the appropriate position in the midway, i.e., $\widetilde{x}_{m}$ in Table 1, to achieve more dexterous welding. In order to accurately locate the starting point of the weld, we define the direction vector $\widetilde{\Gamma}$ to guide the robot to scan the weld (See Table 1). Other parameters are also defined in Table 1.

\subsubsection{Global Positioning of Multiple Welds}

We perform point cloud registration to position the workpiece, and the whole process is illustrated in Fig. 10. In the first step, we sample points from CAD (See Fig. 10 (b)) to form a high-density point cloud which is shown in Fig. 10 (c). Then, we downsample the high-density point cloud to the sparse point cloud, as shown in Fig. 10 (d). We also get the point cloud from RGB-D camera in Fig. 10 (f) after the robot is deployed. Due to the imaging mechanism of the laser speckle of the camera, there may be a large number of point cloud clutter in the field of view, as shown in Fig. 10 (f). Assuming that the actual vertical distance between the workpiece and the optical center of the camera is about $Z_{d}$, with the help of the conditional filtering module in PCL, we filter out

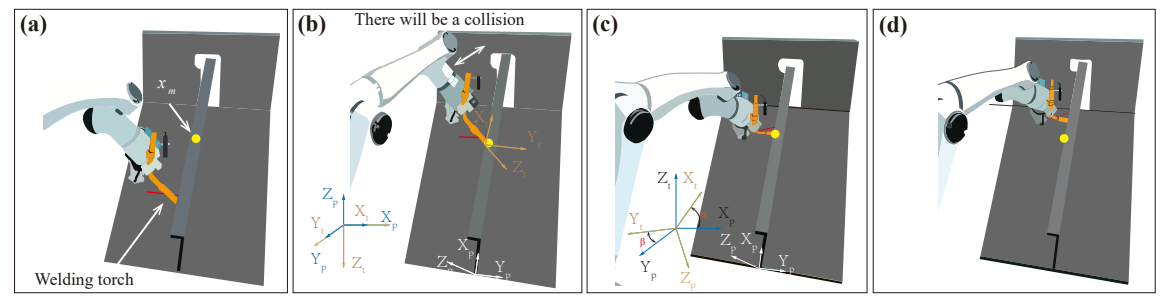

Fig. 9: Dexterous welding of multi-segment welds by adjusting working parameters $\alpha, \beta$ and $d$. (a) A working posture; (b) Collision near the corner along the working posture (a); (c) Adjust the posture parameters $\alpha, \beta$ and $d$ at $\widetilde{x}_{m} ;$ (d) Complete welding near the corner without collisions. 


\section{Table 1: Multiple-weld welding: global parameters.}

$\{M\}$ : Coordinate system of CAD model; $\{B\}$ : Coordinate system of robot base.

\begin{tabular}{ccc}
\hline Feature index & Reference value in $\{M\}$ & Corresponding value in $\{B\}$ \\
\hline Initial point coordinate & $\widetilde{x}_{0}$ & $x_{0}$ \\
End point coordinate & $\widetilde{x}_{1}$ & $x_{1}$ \\
Posture at initial point & $\widetilde{R}_{t}$ & $R_{t}$ \\
Position of changing & $\widetilde{x}_{m}$ & $x_{m}$ \\
working posture & $\widetilde{\Gamma}$ & $\Gamma$ \\
Scanning direction for & & \\
initial point & $\alpha$ & $\alpha$ \\
Working angle & $\beta$ & $\beta$ \\
Traveling angle & $d$ & $d$ \\
Welding pitch & $d$ & \\
\hline
\end{tabular}

the points outside the range of $\left(0-Z_{d}\right)$ in the z-axis direction to obtain clear object point cloud, as the illustrated in Fig $10(\mathrm{~g})$. Correspondingly, we also downsample the captured point cloud to a sparse one in Fig. 10 (h). Finally, we employ ICP to match the two point clouds in Fig 10 (d) and (h), respectively.

\subsubsection{Initial Posture for Different Docking Locations}

Since the position is not necessarily the same at each time it is deployed, that is, the pose between the robot base coordinate system and the workpiece coordinate system is different, if a certain fixed pose is taught, the seam will be out of the range of vision. Therefore, we need to set a proper posture that is fixed to the workpiece to ensure that the robot can correctly image when it reaches the starting point. For any weld, After each deployment of the robot, the following formula holds

$$
\begin{gathered}
{ }^{M} \mathrm{R}_{B}={ }^{M} \mathrm{R}_{C} \cdot{ }^{t} R_{C}^{\mathrm{T}} \cdot{ }^{B} R_{t}^{\mathrm{T}} \\
{ }^{M} R_{t}={ }^{M} \mathrm{R}_{B} \cdot{ }^{B} R_{t}
\end{gathered}
$$

where superscript $B, C, M$ and $t$ represent base coordinate system $\{B\}$, camera coordinate system $\{C\}$, CAD model coordinate system and tool coordinate system $\{t\} .{ }^{t} R_{C}$ is the attitude of the camera coordinate system in the tool coordinate system and it can be obtained by hand-eye calibration. ${ }^{B} R_{t}$ can be queried from the robot controller, and ${ }^{M} R_{C}$ can be obtained by point cloud registration with the help of $3 \mathrm{D}$ camera.

In order to determine the appropriate posture, we adjust the posture of the robot at the starting position in advance that the laser stripe line is approximately in the middle of the image at this pose. We denote the pose of the end-effector of the robot as ${ }^{B} \mathfrak{R}_{t}$, and substitute it into Eq. (22), then the pose ${ }^{M} \mathfrak{R}_{B}$ can be computed. From Eq. (23), we can further obtain the required parameter, denoted as ${ }^{M} \mathfrak{R}_{t}$. Since this attitude is the end-effector relative to the CAD coordinate system, it is independent of the deployment location of 
the robot. For any one deployment of the robot,

$$
{ }^{B} \mathrm{R}_{t}^{i}={ }^{M} \mathfrak{R}_{t} \cdot{ }^{M} \mathrm{R}_{B}^{i}
$$

where the superscript $i$ represents the deployment times. In this way, the relative attitude of the end-effector to the workpiece can be kept unchanged by simply sending ${ }^{B} \mathrm{R}_{t}^{i}$ to the robot controller after each deployment of the robot.

\subsubsection{Positioning of Initial Point}

The automatic guidance of the weld starting point determines the success of the welding. However, at present, the research of initial weld point positioning still remains an open problem [2], and only a few researches about this aspect have been reported. In this paper, we use point cloud registration method to roughly locate the weld, and guide the robot to move to a point which is near the weld starting point. Then we make robot scan the weld so as to accurately locate the starting point. Assuming that coordinates of the initial point of a certain weld in the CAD model is $\widetilde{x}_{0}$, after applying the transformation in (6),
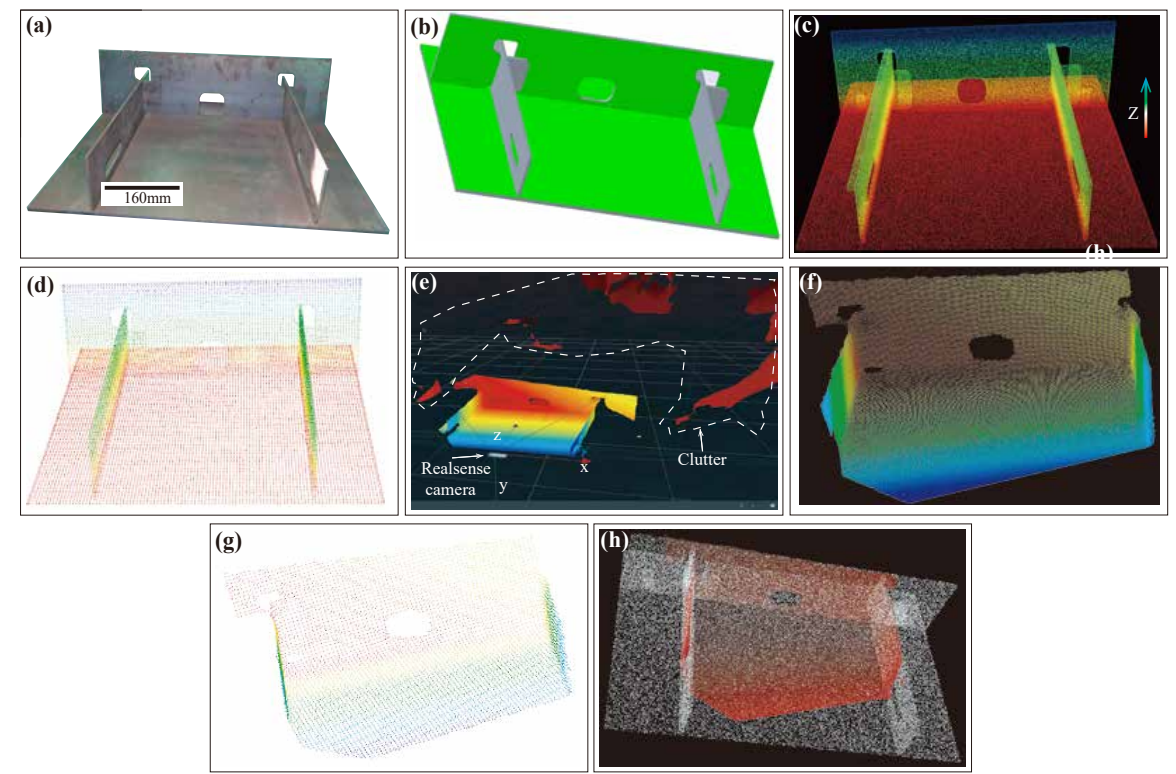

Fig. 10: Multiple-weld positioning via point cloud registration. (a) Original artifact of a ship component; (b) Corresponding CAD model for experiments based on (a); (c) Generated high-density point cloud from (b); (d) Sparse point cloud obtained by voxel grid filtering of (c); (e) Point cloud image of workpiece captured by RGB-D camera. The image is cluttered by other objects; (f) Clutter is filtered by setting the range along $\mathrm{Z}$ direction as well as X direction of (e); (g) The point cloud in (f) is filtered by voxel grid filter and radius outlier filter to obtain the sparse point cloud; (h) Point cloud registration of $(\mathrm{d})$ and $(\mathrm{g})$. 


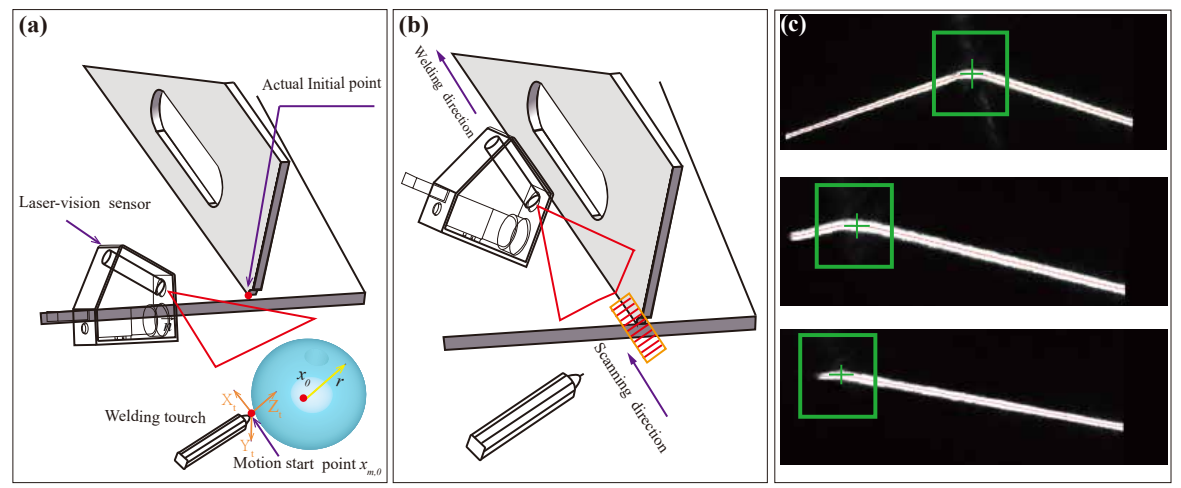

Fig. 11: Accurancy Positioning of initial point. (a) Generate $x_{0}$ via ICP-based positioning of the actual initial point, and drive the robot to $x_{m, 0}$. (b) Scan the starting point via laser-vision sensor and drive the robot move along the defined direction vector $\widetilde{\Gamma}$ on both sides of $x_{0}$. (c) The position of the starting point can be relocated by observing the shape of the laser stripe. We have three possible layer stripe images in the top/middle/bottom to demonstrate three status: robot is far away from/near/at the actual initial point.

we obtain corresponding $x_{0}$ in the robot base coordinate. Directing the robot to the initial point $x_{0}$ may lead to collisions. For this reason, we construct a virtual ball with making $x_{0}$ as the center and $r$ as the radius, so that the actual position of the welding torch falls on the ball sphere, as shown in Fig. 11 (a). Assume that the coordinates of the initial registration point on the sphere is $x_{m, 0}$, it can be determined by the following:

$$
\overrightarrow{O x_{m, 0}}=\overrightarrow{O x_{0}}-r \vec{n}
$$

where $O$ is the origin of $\{B\}$. Give expression for ${ }^{B} \mathrm{R}_{t}^{i}$ in Eq. (24) by ${ }^{B} \mathrm{R}_{t}^{i}=$ $\left.{ }^{B} \widehat{X}_{t},{ }^{B} \widehat{Y}_{t},{ }^{B} \widehat{Z}_{t}\right]$, then $\vec{n}={ }^{B} \hat{Z}_{t}$.

After reaching $x_{m, 0}$, the weld seam is scanned on both sides according to the pre-defined direction $\widetilde{\Gamma}$, and the starting point of the weld seam is accurately approximated by judging the shape of the light line. As shown in Fig. 11 (c), We take the length of laser fringe line on the left as the basis for judgment. When the welding torch gradually approaches the starting point of the weld, the period length decreases. When its length is less than a certain threshold value, it can be considered to have reached the starting point of the weld.

\section{Experiments}

To verify the feasibility of the proposed seam-tracking system, three types of experiments are carried out. The purpose of the first experiment is to verify the accuracy of our laser-vision sensor; the second experiment is to confirm the 
effects of the single-weld seam tracking, and the last one is to test the performance of multiple-weld seams tracking with considering changeable elements under unstructured environments. In the test of single-weld seam tracking, instead of choosing a real torch with a welding wire as the end-effector, we adopt a displacement sensor with a rigid pen as the experimental torch, see Fig. 13 (a) and 14 (a). Compared to the deformable welding wire, it becomes easier to observe the subtle collision and interference between the end-effector and the workpiece.

After testing our tracking method for a single weld, we used a ship structure as a test artifact to verify the feasibility of the continuous tracking of multiple welds. Since the difference between the multiple-weld and single-weld tracking is that the multiple-weld tracking requires the global positioning of the artifact, in this section, we focus on verifying the system performance in terms of the global positioning in an unstructured environment. The experimental platform for the case of multiple-weld tracking is shown in Fig. 16.

\subsection{Precision Evaluation}

Based on the mathematical model in Eq. 5 of Section 2.3, we can roughly obtain the three-dimensional coordinate $P_{i}=\left[X_{c}, Y_{c}, Z_{c}\right]^{T}$ of a point $P_{i}$ in the camera coordinate system through the 2-dimensional pixel coordinate $p_{i}=$ $[u, v]^{T}$ in image coordinate system. In order to facilitate the operation, we choose to indirectly reflect the situation of coordinate measurement via the distance between two points. As shown in Fig. 12 (a), we keep the laser vision sensor stationary and place the calibration plate in an appropriate position so that the edge of a row of the checkerboard grid coincides with the laser line. We evaluate the accuracy of our model by measuring the sides of the squares. The side length of each square shown in Fig. 12 (a) is $7 \mathrm{~mm}$, and the manufacturing error is only $\pm 0.01 \mathrm{~mm}$. For a certain grid in a row, it is assumed that the coordinates of the left and right corner points are ${ }^{c} \mathrm{P}_{i, L}=\left[x_{i, L}, y_{i, L}, z_{i, L}\right]^{T}$ and
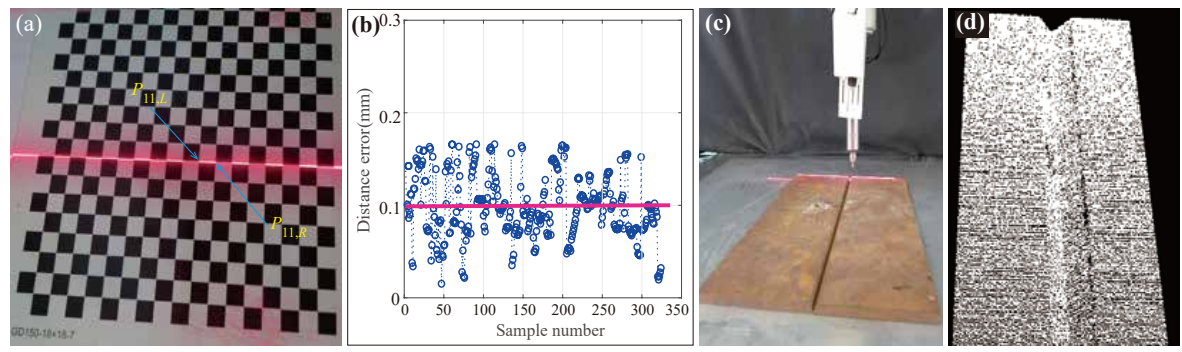

Fig. 12: Precision evaluation of the 3D reconstructed model. (a)The measuring accuracy of the mathematics model is verified by the square length in the calibration plate; (b) The deviation range of all grid measurements; (c) A straight weld seam to be scanned; (d) Weld point cloud obtained by 3D reconstruction of the weld after scanning. 


\section{Springer Nature $2021 \mathrm{LAT}_{\mathrm{E}} \mathrm{X}$ template}

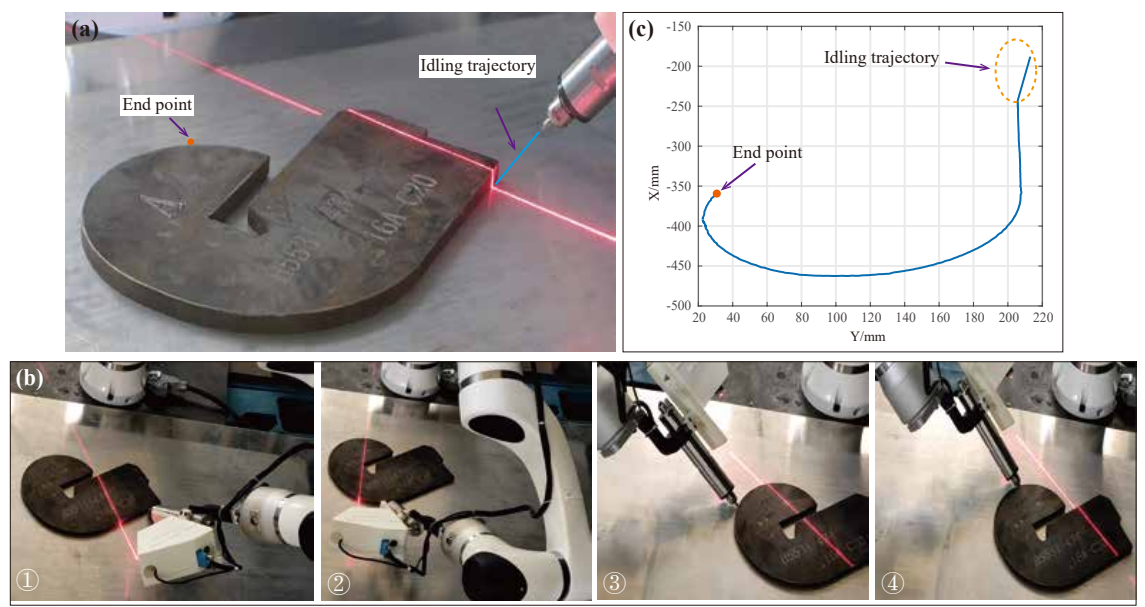

Fig. 13: No prior information is used for tracking large curvature complex welds. (a) Experimental scene; (b)Snapshots during the tracking process. (c) Tracking trajectory of robot end-effector;

${ }^{c} \mathrm{P}_{i, R}=\left[x_{i, R}, y_{i, R}, z_{i, R}\right]^{T}$ respectively. We define the measurement error as:

$e_{i}=\left\|P_{i, L}-P_{i, R}\right\|_{2}-7=\sqrt{\left(x_{i, L}-x_{i, R}\right)^{2}+\left(y_{i, L}-y_{i, R}\right)^{2}+\left(z_{i, L}-z_{i, R}\right)^{2}}-7$

We measure each of 324 squares on the calibration board and calculate their measurement errors. The result obtained is shown in Fig. 12 (b). The experiment shows that the average measurement error is $\widetilde{E}=0.0991 \mathrm{~mm}$, and the overall deviation range could be controlled to be within $0.1659 \sim 0.0152 \mathrm{~mm}$, using our calibration method in Section 2.3.2. To further verify the 3D reconstruction capability of the designed sensor, we perform 3D reconstruction of all the pixel points on the whole laser stripe. As shown in Fig. 12 (c), we make the robot carry the sensor along a fixed route and quickly scan a straight weld seam. The obtained result is shown in Fig. 12 (d). It can be seen from the figure that our reconstructed data points can clearly reflect the 3D shape of the weld seam.

\subsection{Single-Weld Tracking}

\subsubsection{Seam Tracking of a Complex Space Curve}

To verify the tracking method proposed in this work, a special shaped part with large curvature is selected for experiment. As shown in Fig. 13 (a), the part is placed on the working platform. After the starting position is given, the robot first moves to the starting point. Once it reaches the starting point, the end-effector of the robot starts the adaptive attitude adjustment which is to determine the autonomous movements along the curved weld until it reaches the given end point. Part of photos during the tracking process are shown in Fig. 13 (c). The whole tracking process does not need any manual intervention 
or prior information like the curve equation of the weld seam, the CAD model, and the pre-taught trajectory, etc. While the robot is tracking along the weld seam, we record the position of the robot end-effector at each unit moment in the backend program of the host computer. Projecting these position points to the $X O Y$ plane of the robot base coordinate frame, the recorded curve is shown in Fig. 13 (b). It can be seen that the motion trajectory of the robot is highly matching the weld profile of the part.

\subsubsection{Accuracy Evaluation of Single-Weld Tracking}

The basic principle of the precision evaluating for the weld seam tracking is to compare the moving trajectory of the welding torch with the actual weld seam trajectory. However, it is a difficult task to precisely obtain the trajectory distribution of the actual curved weld. Particularly, the true value of the coordinates of the points on the weld is hard to obtain. For this reason, most of the current practices are to sample the weld seam points through teaching method to obtain true coordinates of multiple trajectory points, such as the method in the experiments of these works [16, 26, 27]. In order to more accurately compare the difference between two trajectories, we first calibrate the position and the pose relationship between the coordinate system of the workpiece and the robot base coordinate system. The purpose is to convert the coordinates of the points on the weld to the base coordinate frame of the robot, as shown in Fig. 14 (c). The established workpiece coordinate system is shown in Fig. 14 (a). Coordinates of each point on the weld are extracted after establishing the same coordinate system on the CAD model of the workpiece
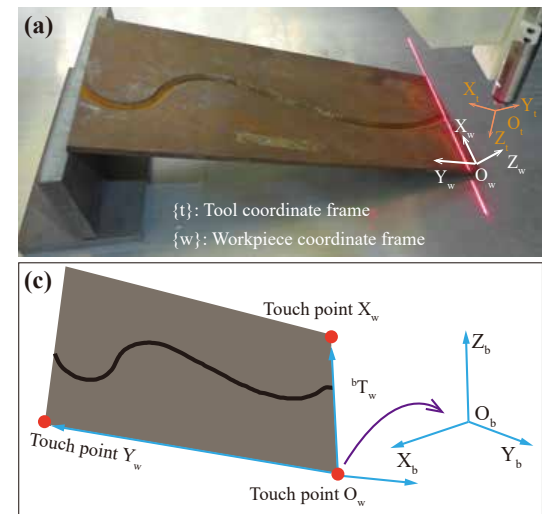
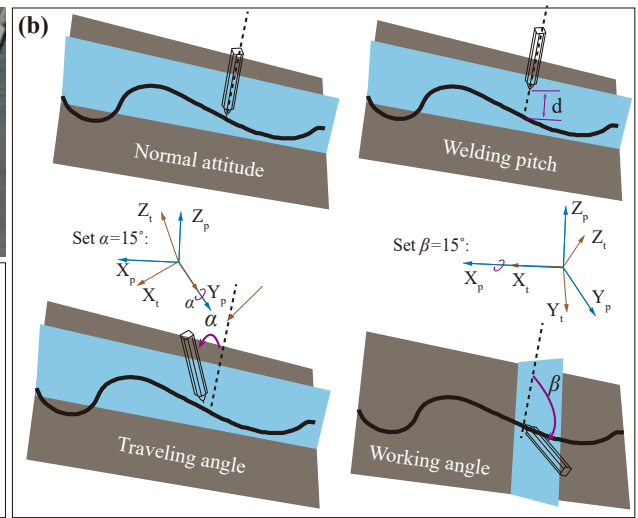

Fig. 14: Verification of the effectiveness of welding seam tracking under different working parameters. (a) The space weld to be tested and the associated coordinate system; (b) Four types of tests were carried out for different working parameters; (c) By converting the workpiece coordinate system to the robot base coordinate system, we can obtain the trajectory distribution of the weld to be tested in the robot base coordinate system. 
to obtain the actual trajectory distribution of the weld. Since we strictly control the manufacturing errors of the workpiece, it is therefore able to mainly ensure the consistency of the distribution of the actual weld seam and the seam trajectory on CAD. Assume that the coordinate of a point $P_{i}$ on the weld under the workpiece coordinate system, it can be converted to the base coordinate system by the following equation:

$$
{ }^{b} P_{i}={ }^{b} T_{w} \cdot{ }^{w} P_{i}
$$

By applying Eq. (27) to all points on the weld, the trajectory distribution of the actual weld in the robot base coordinate system can be obtained.

The accuracy evaluation experiments are divided into four groups, as shown in Fig. 14 (b), i.e., the weld tracking with different working parameters in Fig. 14 (a). In terms of the trajectory accuracy evaluation, we accurately compare the recorded robot trajectory with the actual welding seam trajectory. After tracking with normal attitude, we plot the motion trajectory of robot and the actual weld position in the same diagram, as shown in Fig. 15 (a). It can be clearly seen that the two trajectories are highly consistent. In the experiment of setting welding pitch, Fig. 15(b) shows the recorded robot motion trajectory with setting $d=5 \mathrm{~mm}$. It can be seen that the robot motion trajectory is almost parallel to the actual weld trajectory, and the spacing of two trajectories is basically the same. In order to evaluate the consistency of the trajectory quantitatively, for any point $\widetilde{P}_{i}(x, y, z)$ on the motion trajectory of the end-effector, the nearest Euclidean point $P_{i}(x, y, z)$ is taken as its corresponding weld trajectory point, and the Euclidean distance $e_{i}=\left\|\widetilde{P}_{i}-P_{i}\right\|_{2}=\sqrt{\left(\widetilde{x_{i}}-x_{i}\right)^{2}+\left(\widetilde{y_{i}}-y_{i}\right)^{2}+\left(\widetilde{z_{i}}-z_{i}\right)^{2}}$ between two points is
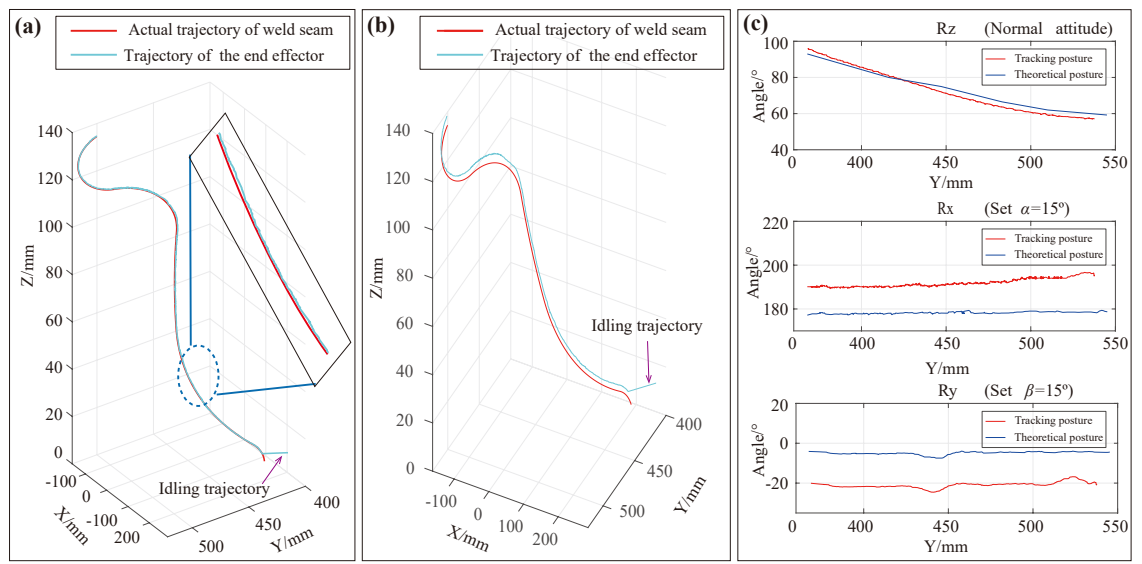

Fig. 15: Trajectory comparison results of the experiment in Fig. 14. (a) Robot motion trajectory and weld seam trajectory of the normal attitude experiment in Fig. 14 (b); (b) Change of angle of robot tool coordinate system after changing working angle and travel angle; (c) The result of the changing welding pitch experiment in Fig. 14 (b). 
Table 2: Trajectory error of experiment in Fig. 14 (b).

\begin{tabular}{llcccc}
\hline Experiment Group & $\begin{array}{l}\text { Sample points } \\
\text { numbe }\end{array}$ & $\begin{array}{c}\text { Max } \\
\text { error/mm }\end{array}$ & $\begin{array}{c}\text { Mean } \\
\text { error/mm }\end{array}$ & Min error/mm RMSE/mm \\
\hline Normal attitude & 6050 & 0.6421 & 0.4526 & $2.405 \times 10^{-5}$ & 0.1975 \\
Welding pitch & 6397 & 0.8521 & 0.5663 & $1.193 \times 10^{-4}$ & 0.3595 \\
Traveling angle & 6119 & 0.7996 & 0.3972 & $1.794 \times 10^{-4}$ & 0.2271 \\
Working angle & 6278 & 0.6970 & 0.3564 & $9.800 \times 10^{-3}$ & 0.1843 \\
\hline
\end{tabular}

Table 3: Attitude error of experiment in Fig. 14(b)

\begin{tabular}{lllll}
\hline Experiment Group & Max error $\left(^{\circ}\right)$ & Mean error $\left(^{\circ}\right)$ & Min error $\left(^{\circ}\right)$ & $\operatorname{RMSE}\left({ }^{\circ}\right)$ \\
\hline$R_{Z}$ & 1.865 & 0.9614 & 0.6903 & 0.4767 \\
$R_{X}$ & 3.237 & 2.958 & 2.029 & 0.5004 \\
$R_{Y}$ & 4.151 & 3.627 & $6.894 \times 10^{-4}$ & 0.9419 \\
\hline
\end{tabular}

regarded as the trajectory tracking error. The error statistics of the 4 groups of experiments are shown in Table. 2. For the evaluation of the tracking attitude, the theoretical tracking posture is obtained by the teaching method and saved for subsequent deviation comparisons. As illustrated in Fig. 14 (b), when evaluating the tracking attitude of the normal attitude, we have $\alpha$ and set $\beta$ experiments. We select the attitude angles of the robot's $\mathrm{Z}$ axis, $\mathrm{X}$ axis and $\mathrm{Y}$ axis respectively as the reference, and Fig. 15 (b) shows corresponding recorded values of these three angles. Table 3 shows the analysis of the posture error during the tracking process. From the experimental results, we can see that the robot can basically moves along the actual weld trajectory according to the desired attitude with the adjusted working parameters.

\subsection{Multiple-Weld Tracking}

It has been known that under an unstructured environment, the robot might face different lighting conditions, which often affects the image quality, and thus affects the positioning performance. In order to test the global positioning performance of the system under different illuminations, we carried out experiments under different values of illumination. The test environments are divided into indoor environment and outdoor environment (see Fig. 16). In the indoor environment, we use an adjustable light source to generate different lighting conditions. We take an easy-to-measure end point in the structural part as a reference point, as shown in Fig. 16 (b). Under a certain lighting condition, after the end-effector of robot moves to the reference point in a certain positioning posture according to the method in Section 3.2.1, a vernier caliper was used to measure the distance $d$ between the end of the torch and the reference point, and this distance was regarded as the positioning error of the system. Keep the lighting conditions unchanged, and change the placement position of the robot again to measure the positioning result of the robot. This experiment is repeated for 20 times, and the positioning errors of the robot are recorded as the final positioning errors under a certain illumination 
condition. For example, as shown in Fig. 17 (a), the first group of data is the statistical result of positioning errors from 20 groups under the 315LX illumination. In Fig. 17 (a), the $1789 \sim 81400$ LX illumination data is from the outdoor environment test. The test location is in the outdoor environment on a sunny summer day and the continuous test time is between 13:00 and 18:00 p.m. It can be observed from this figure that even under the condition of the strong sunlight, the error of the positioning via ICP is basically in between 10$25 \mathrm{~mm}$, which indicates that even if the robot is placed in different places, our method can guide the robot to reach the vicinity of the starting point of the weld. We conducted a tracking test on the multi-segment welds in the experimental structure in outdoor environment. The results show that the robot can track the multi-segment welds smoothly without any human intervention in the middle. The recorded tracking trajectory is shown in the Fig. 17 (b).

Overall, in terms of space welds, the proposed algorithm has better flexibility than the traditional Teaching-playback working modes of manipulators. Due to the good lightness of collaborative manipulator, this makes it easy to deploy. By human-machine collaboration, we can easily adjust the photo posture of the $3 \mathrm{D}$ camera to globally position the multi-seams in the workpiece, even if it is placed in a different position. We first performed experimental tests on a single weld under a variety of conditions, and then performed

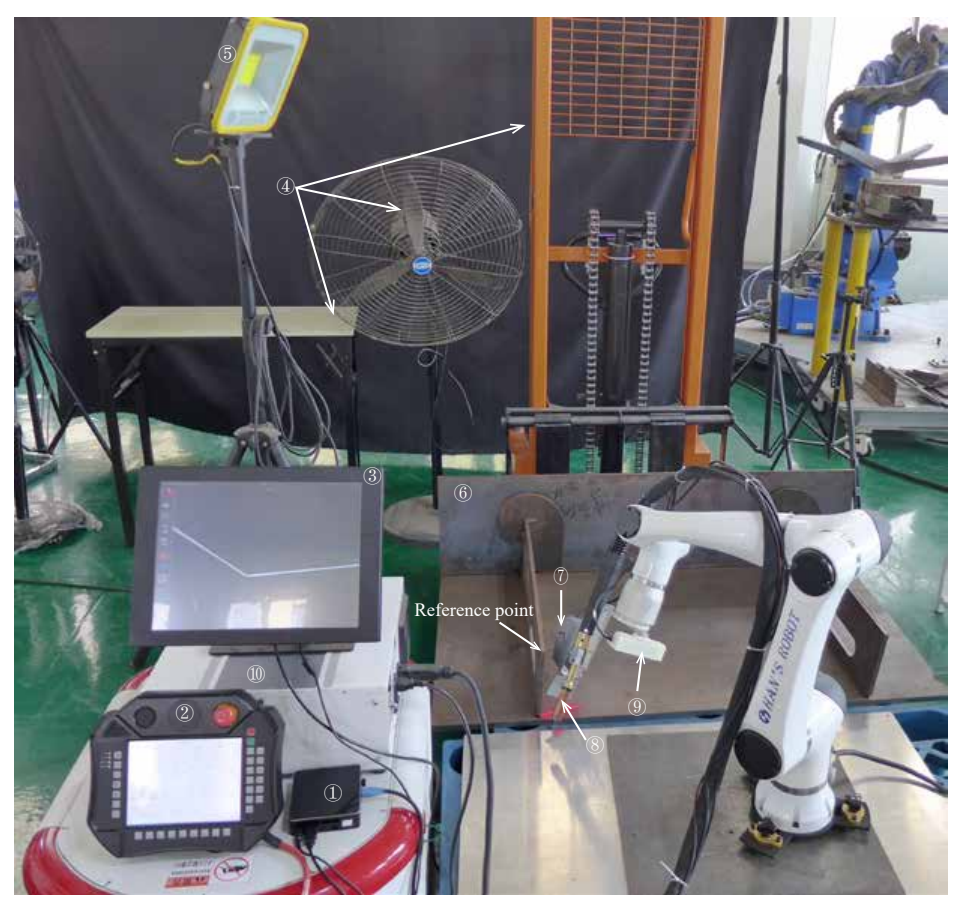

Fig. 16: Experimental workspace. (1)Host computer (2)Teaching pendant (3) Touch-screen computer (4)Background interferers (5)Fluorescent lamp (6)Workpiece (7Laser-vision sensor 8Welding torch (9RGB-D camera (10Robot controller; (b) Outdoor experimental workspace. 

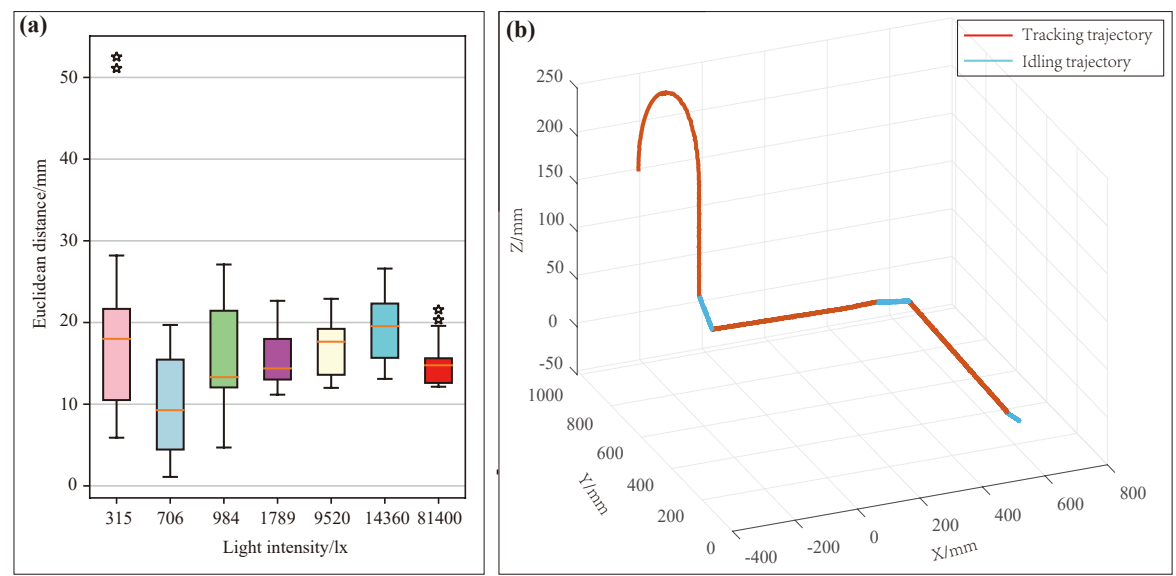

Fig. 17: System global positioning performance test under different deployment location and environmental conditions. (a) Global positioning performance under different illumination; (b) Motion trajectory of the robot for tracking multiple weld seams.

autonomous welding tests on multiple welds in the workpiece, and both obtained satisfactory results.

\section{Conclusion}

In this paper, we demonstrate an autonomous tracking method for multisection space welds. The practical experiments show that the tracking performance is excellent. Based on the visual guidance method, we propose a dual sequence tracking mechanism, which can effectively solve the problem that traditional methods cannot flexibly adjust important welding parameters (e.g, the working angle and the traveling angle). Via point cloud registration for global positioning of the workpiece, the autonomous tracking of multi-segment welds can be realized. Using collaborative robots to form a portable automatic welding system, our research could better fix the problem of autonomous tracking of spatial welds under an unstructured environment. Our method provides the possibility of fully autonomous robot welding under unstructured enviroment in the future.

Author contribution Shuangfei Yu: conceptualization, investigation, methodology, and writing original draft preparation.

Yisheng Guan: project leader,investigation and validation.

Zhi Yang:partial methodology, experimental assistance.

Chutian Liu: experimental assistance.

Jiacheng Hu: experimental assistance.

Jie Hong: writing and original draft preparation .

Haifei Zhu: investigation and original draft preparation.

Tao Zhang: original draft preparation and validation. 
Funding This work was supported in part by the Research and Development Programs in Key Areas of Guangdong Province (Grant No.2020B090928002), the Key RD Program of Guangdong Province (Grant No. 2019B090915001), Natural Science Foundation of China (Grant No. 51905105), the Natural Science Foundation of Guangdong Province (Regular Project: Grant No. 2020A1515011262), and the State Key Laboratory of Robotics and Systems (HIT) (Grant No. SKLRS-2020-KF12).

Data availability Not applicable.

Code availability Not applicable.

\section{Declarations}

Ethics approval Not applicable.

Consent for publication All authors have read and agreed to the published version of the manuscript.

Competing interests The authors declare no competing interests. 


\section{References}

[1] Nikolov, M.: Trends in development of weld overlaying during the 21 century. Acta technologica agriculturae 17(2), 35-38 (2014)

[2] Fang, Z., Xu, D., Tan, M.: Vision-based initial weld point positioning using the geometric relationship between two seams. The international journal of advanced manufacturing technology 66(9-12), 1535-1543 (2013)

[3] Xu, Y., Yu, H., Zhong, J., Lin, T., Chen, S.: Real-time seam tracking control technology during welding robot gtaw process based on passive vision sensor. Journal of Materials Processing Technology 212(8), 1654$1662(2012)$

[4] Chen, X., Dharmawan, A.G., Foong, S., Soh, G.S.: Seam tracking of large pipe structures for an agile robotic welding system mounted on scaffold structures. Robotics and Computer-Integrated Manufacturing 50, 242255 (2018)

[5] Liu, W., Du, X., Chen, H., Sun, H.: Visual feature extraction and tracking control for narrow seam in three dimension space. In: Proceeding of the 11th World Congress on Intelligent Control and Automation, pp. 55565560 (2014). IEEE

[6] Li, G., Hong, Y., Gao, J., Hong, B., Li, X.: Welding seam trajectory recognition for automated skip welding guidance of a spatially intermittent welding seam based on laser vision sensor. Sensors 20(13), 3657 (2020)

[7] Qi, R., Zhou, W., Zhang, H., Zhang, W., Yang, G.: Trace generation of friction stir welding robot for space weld joint on large thin-walled parts. Industrial Robot: An International Journal (2016)

[8] Yanfeng, G., Hua, Z., Yanhui, Y.: Back-stepping and neural network control of a mobile robot for curved weld seam tracking. Procedia Engineering 15, 38-44 (2011)

[9] Feng, X., Gao, L., Tian, W., Wei, R., Wang, Z., Chen, Y.: Application of wall climbing welding robot in automatic welding of island spherical tank. Journal of Coastal Research 107(SI), 1-4 (2020)

[10] Guo, J., Zhu, Z., Sun, B., Zhang, T.: A novel field box girder welding robot and realization of all-position welding process based on visual servoing. Journal of Manufacturing Processes 63, 70-79 (2021)

[11] Tool, I.I., Sales, M.: ITM "LIZARD" WELDING CARRIAGE. https://www.industrialtool.com.au/product/ 2831-itm-lizard-welding-carriage-240v-horizontal-0-1200mm-min? 
categoryId=838/. November 10,2021

[12] Koike Aronson, I.: Wel-Handy Multi Next. https://www.koike.com/ wel-handy-multi-next. November 10, 2021

[13] Park, J.-H., Moon, H.-S.: Advanced automatic welding system for offshore pipeline system with seam tracking function. Applied Sciences 10(1), 324 (2020)

[14] Ku, N., Cha, J.-h., Lee, K.-Y., Kim, J., Kim, T.-w., Ha, S., Lee, D.: Development of a mobile welding robot for double-hull structures in shipbuilding. Journal of marine science and technology 15(4), 374-385 (2010)

[15] Kim, J., Lee, J., Chung, M., Shin, Y.-G.: Multiple weld seam extraction from rgb-depth images for automatic robotic welding via point cloud registration. Multimedia Tools and Applications 80(6), 9703-9719 (2021)

[16] Shah, H.N.M., Sulaiman, M., Shukor, A.Z.: Autonomous detection and identification of weld seam path shape position. The International Journal of Advanced Manufacturing Technology 92(9), 3739-3747 (2017)

[17] Ding, Y., Huang, W., Kovacevic, R.: An on-line shape-matching weld seam tracking system. Robotics and Computer-Integrated Manufacturing 42, 103-112 (2016)

[18] Dinham, M., Fang, G.: Autonomous weld seam identification and localisation using eye-in-hand stereo vision for robotic arc welding. Robotics and Computer-Integrated Manufacturing 29(5), 288-301 (2013)

[19] Baek, D., Moon, H.S., Park, S.-H.: Development of an automatic orbital welding system with robust weaving width control and a seam-tracking function for narrow grooves. The International Journal of Advanced Manufacturing Technology 93(1), 767-777 (2017)

[20] Du, R., Xu, Y., Hou, Z., Shu, J., Chen, S.: Strong noise image processing for vision-based seam tracking in robotic gas metal arc welding. The International Journal of Advanced Manufacturing Technology 101(5), $2135-2149(2019)$

[21] Muhammad, J., Altun, H., Abo-Serie, E.: Welding seam profiling techniques based on active vision sensing for intelligent robotic welding. The International Journal of Advanced Manufacturing Technology 88(1-4), 127-145 (2017)

[22] Banafian, N., Fesharakifard, R., Menhaj, M.B.: Precise seam tracking in 
robotic welding by an improved image processing approach. The International Journal of Advanced Manufacturing Technology 114(1), 251-270 (2021)

[23] Zhao, Z., Luo, J., Wang, Y., Bai, L., Han, J.: Additive seam tracking technology based on laser vision. The International Journal of Advanced Manufacturing Technology, 1-15 (2021)

[24] Nele, L., Sarno, E., Keshari, A.: An image acquisition system for real-time seam tracking. The International Journal of Advanced Manufacturing Technology 69(9-12), 2099-2110 (2013)

[25] Shi, F., Zhou, L., Lin, T., Chen, S.: Efficient weld seam detection for robotic welding from a single image. In: Robotic Welding, Intelligence and Automation, pp. 289-294 (2007). Springer

[26] Zou, Y., Chen, J., Wei, X.: Research on a real-time pose estimation method for a seam tracking system. Optics and Lasers in Engineering 127, 105947 (2020)

[27] De Graaf, M., Aarts, R., Jonker, B., Meijer, J.: Real-time seam tracking for robotic laser welding using trajectory-based control. Control engineering practice 18(8), 944-953 (2010)

[28] Xu, Y., Lv, N., Fang, G., Du, S., Zhao, W., Ye, Z., Chen, S.: Welding seam tracking in robotic gas metal arc welding. Journal of Materials Processing Technology 248, 18-30 (2017)

[29] Moon, H.-S., Ko, S.-H., Kim, J.-C.: Automatic seam tracking in pipeline welding with narrow groove. The International Journal of Advanced Manufacturing Technology 41(3-4), 234-241 (2009)

[30] Regaard, B., Kaierle, S., Poprawe, R.: Seam-tracking for high precision laser welding applications - methods, restrictions and enhanced concepts. Journal of Laser Applications 21(4), 183-195 (2009)

[31] Henriques, J.F., Caseiro, R., Martins, P., Batista, J.: High-speed tracking with kernelized correlation filters. IEEE Transactions on Pattern Analysis and Machine Intelligence 37(3), 583-596 (2015). https://doi.org/10.1109/ TPAMI.2014.2345390

[32] Corke, P.: Robotics, Vision and Control: Fundamental Algorithms in MATLABß Second, Completely revised vol. 118, (2017). Springer

[33] Yu, S., Hong, J., Zhang, T., Yang, Z., Guan, Y.: A self-correction based algorithm for single-shot camera calibration. In: International Conference on Intelligent Robotics and Applications, pp. 442-455 (2020). Springer 
[34] Idrobo-Pizo, G.A., Motta, J.M.S., Sampaio, R.C.: A calibration method for a laser triangulation scanner mounted on a robot arm for surface mapping. Sensors 19(8), 1783 (2019)

[35] Yanbiao, Zou, Xiangzhi, Chen: Hand-eye calibration of arc welding robot and laser vision sensor through semidefinite programming. Industrial Robot: An International Journal 45(5), 597-610 (2018)

[36] Xiao, R., Xu, Y., Hou, Z., Chen, C., Chen, S.: An automatic calibration algorithm for laser vision sensor in robotic autonomous welding system. Journal of Intelligent Manufacturing, 1-14 (2021)

[37] Li, J., Li, H., Wei, H., Gao, Y.: Effect of torch position and angle on welding quality and welding process stability in pulse on pulse mig welding-brazing of aluminum alloy to stainless steel. The International Journal of Advanced Manufacturing Technology 84(1-4), 705-716 (2016)

[38] Yu, J., Kim, D.: Effects of welding current and torch position parameters on minimizing the weld porosity of zinc-coated steel. The International Journal of Advanced Manufacturing Technology 95(1), 551-567 (2018)

[39] Teeravarunyou, S., Poopatb, B.: Computer based welding training system. International Journal of Industrial Engineering 16(2), 116-125 (2009)

[40] Shultz, E.F., Cole, E.G., Smith, C.B., Zinn, M.R., Ferrier, N.J., Pfefferkorn, F.E.: Effect of compliance and travel angle on friction stir welding with gaps. Journal of manufacturing science and engineering 132(4) (2010)

[41] Besl, P.J., Mckay, H.D.: A method for registration of 3-d shapes. IEEE Transactions on Pattern Analysis \& Machine Intelligence 14(2), 239-256 (1992) 